\title{
Topography of Bone Erosions at the Metatarsophalangeal Joints in Rheumatoid Arthritis: Bilateral Mapping by Computed Tomography
}

Paolo Simoni ${ }^{1}$, Sakina Moussaddykine ${ }^{1}$, Olivier Malaise ${ }^{2}$, Selma Ben Mustapha ${ }^{3}$, Maria Pilar Aparisi Gómez ${ }^{4,5}$, Alessandro De Leucio ${ }^{6}$

1. Radiology, Queen Fabiola Children's University Hospital - Université Libre de Bruxelles, Brussels, BEL 2. Rheumatology, Centre Hospitalier Universitaire de Liège - Université de Liège, Liège, BEL 3. Radiation Oncology, Centre Hospitalier Universitaire de Liège - Université de Liège, Liège, BEL 4. Radiology, Auckland City Hospital, Auckland, NZL 5. Radiology, Hospital Vithas Nueve De Octubre, Valencia, ESP 6. Radiology and Medical Imaging, Queen Fabiola Children's University Hospital, Brussels, BEL

Corresponding author: Paolo Simoni, paoloemiliosimoni@gmail.com

\section{Abstract}

Objectives: To describe the bilateral anatomical location of bone erosions (BE) at the metatarsophalangeal joints in patients with rheumatoid arthritis using computed tomography.

Materials and methods: Eighteen consecutive patients with established rheumatoid arthritis prospectively underwent computed tomography of both forefeet. Each joint surface of the metatarsal heads (MTH) and the proximal phalangeal bases were divided into four quadrants: superior, plantar, tibial, and fibular. The number of BE was cumulatively counted per patient, side, joint, per joint surface, and quadrant. Descriptive statistics, paired and unpaired samples t-tests, Pearson's correlation coefficients, ANOVA 2, and variance component analysis were performed.

Results: There were $288 \mathrm{BE}$ at the MTH and 66 at the proximal phalanges. The number of BE in one forefoot was a poor predictor of the absolute number of $\mathrm{BE}$ on the contralateral foot " $\mathrm{r}=0.54$ " and was unrelated to symptoms.

The superior quadrants were less frequently affected than other quadrants for both the MTH "p<0.0001" and proximal phalanges " $\mathrm{p}<0.001$." The tibial quadrant showed a higher number of $\mathrm{BE}$ compared to all other quadrants for MTH " $p<0.03$," proximal phalanges " $p<0.01$, and for the metatarsophalangeal joint as a whole "p<0.0001." Plantar and fibular quadrants were equally affected "p<0.05."

Conclusion: BE were found more frequently on the tibial side of the MTH in patients with rheumatoid arthritis.

Review began 06/01/2021 Review ended 06/09/2021 Published 06/22/2021

\section{() Copyright 2021}

Simoni et al. This is an open access article distributed under the terms of the Creative Commons Attribution License CC-BY 4.0., which permits unrestricted use, distribution, and reproduction in any medium, provided the original author and source are credited.
Categories: Radiology, Rheumatology

Keywords: rheumatoid arthritis, erosion, metatarsophalangeal joint, computed tomography, mri

\section{Introduction}

Rheumatoid arthritis (RA) is a chronic inflammatory disease causing joint swelling, tenderness, chronic disability, and reduced life expectancy [1]. RA has a worldwide prevalence of $0.5-1.0 \%$ [2]. Most RA patients are diagnosed between the age of 35 and 50 years [2]. Bone erosions (BE) play a key role in joint damage, causing intra-articular bone loss and joint failure [3].

The metatarsophalangeal joints (MTPJ) are the most affected anatomical areas of the lower feet [4]. In $57 \%$ of patients with established RA, the number of BE at the MTPJ on radiographs is directly related to the degree of walking limitation and the long-term quality of life [5]. As in other joints, in the MTPJ, BE were classically associated with the presence of the synovial pannus penetrating the subchondral bone at the so-called joint "bare areas" [6]. However, the advent of MRI highlighted the importance of the inflammatory edema of the subchondral bone marrow in the pathogenesis of BE [7]. However, as proven in different joints, neither synovial pannus nor subchondral bone edema is spatially related to the specific distribution of the $\mathrm{BE}[8]$.

In 1965, Martel et al. [9] first suggested that BE might be more frequent in joint areas exposed to higher mechanical demand. More recently, Siddle et al. [10] demonstrated that BE were statistically more frequently found at the plantar aspect of the metatarsal heads (MTH) on the MRI assessment limited to the more symptomatic forefoot. These findings support the hypothesis that mechanical demand for the BE can determine the BE location at the MTPJ. However, this study was limited to the more symptomatic side. 
Computed tomography (CT) enables a fast and low-dose examination of both forefeet in the same patients [11]. In addition, CT, due to its spatial resolution and its exquisite capability to assess the mineral structure of the bone, is considered the gold standard imaging technique to detect BE in clinical settings [11-14].

This study aims to provide a first description of the bilateral anatomical distribution of BE at the MTPJ using computed tomography to gauge the hypothesis that mechanical demand determines their distribution.

\section{Materials And Methods \\ Study population and image collection}

We retrospectively reviewed the CT images of both forefeet of 18 consecutive outpatients referred to the Rheumatology Department of the University Hospital of Liège, Belgium, from March to May 2014. This population was assessed by CT to compare the accuracy of tomosynthesis with that of X-rays, using CT as the gold standard in a previously published prospective study [11]. All patients enrolled met the American College of Rheumatology/European League Against Rheumatism criteria for RA [1]. All subjects were older than 18 years. All patients had established RA, defined as RA with a diagnosis older than six months. To describe the anatomical location of BE of both feet, CT images were retrieved from the local Picture Archiving and Communicating System (PACS). The CT images were reviewed by one musculoskeletal radiologist (PS) with 16 years of experience, blinded to the demographic and clinical data.

The Local Ethics Committee approved this retrospective study, and patients' written consensus was waived for this retrospective study.

\section{CT technique}

CT examinations were performed using a 16-slice multidetector CT (Sensation 16, Siemens, Erlangen, Germany). Patients were positioned supine with ankles in slight extension, the heels resting on the table, and the feet placed on a cushion. Feet were scanned from the ankle joint to the toe tips. Native images were obtained in a "feet first and prone" position, the ankle forming a $90^{\circ}$ angle with the legs. A standard protocol using $120 \mathrm{kV}$, an effective tube current-time product of $108 \mathrm{mAs}$, a pitch of 0.5 , and a collimation of $6 \times 0.75 \mathrm{~mm}^{2}$ was used. Native slices of $0.75 \mathrm{~mm}$ were obtained using the U70 Kernel. The mean dose-length product calculated on population was $206.3 \pm 24.2 \mathrm{mGy}{ }^{*} \mathrm{~cm}$.

\section{Study population and image collection}

All images were anonymised and reviewed using a medical workstation (IMPAX, Agfa HealthCare, version 6.5.3.1509, Mortsel, Belgium) equipped with a pair of 5-megapixel medical screens (MDCC-6230, Barco, Kortrijk, Belgium with a physical size of $654 \times 409 \mathrm{~mm}^{2}$ and a native resolution of $3280 \times 2048$ pixels). Multiplanar reconstruction (MPR) images in all required plans were performed.

MPR images were manually obtained by the reviewing radiologist, using the Average Intensity Projection and a slice thickness of $2 \mathrm{~mm}$. The window was set with window level centred at 330 Hounsfield Unit (HU) and window width of $1400 \mathrm{HU}$.

\section{Operational definition}

Bone Erosions

According to the international recommendations [7], BE was defined as a bony defect in the joint or an abrupt loss of the subchondral bone plate on at least two planes on CT images on MPR.

BE Topography

Each joint surface, including the MTH and the bases of the proximal phalanges (PP), was divided into four quadrants, including a superior (dorsal), inferior (plantar), medial (tibial), and lateral (fibular) quadrants, according to previous studies (Figures 1 and 2) $[8,15]$. This joint mapping allows separating the plantar quadrant of the joint subjected to the weight of the body in standing position from the tibial (i.e., medial) and the fibular (i.e., lateral) quadrants where the mechanical demand is elicited by the entheses of the collateral ligaments [8]. 


\section{Cureus}

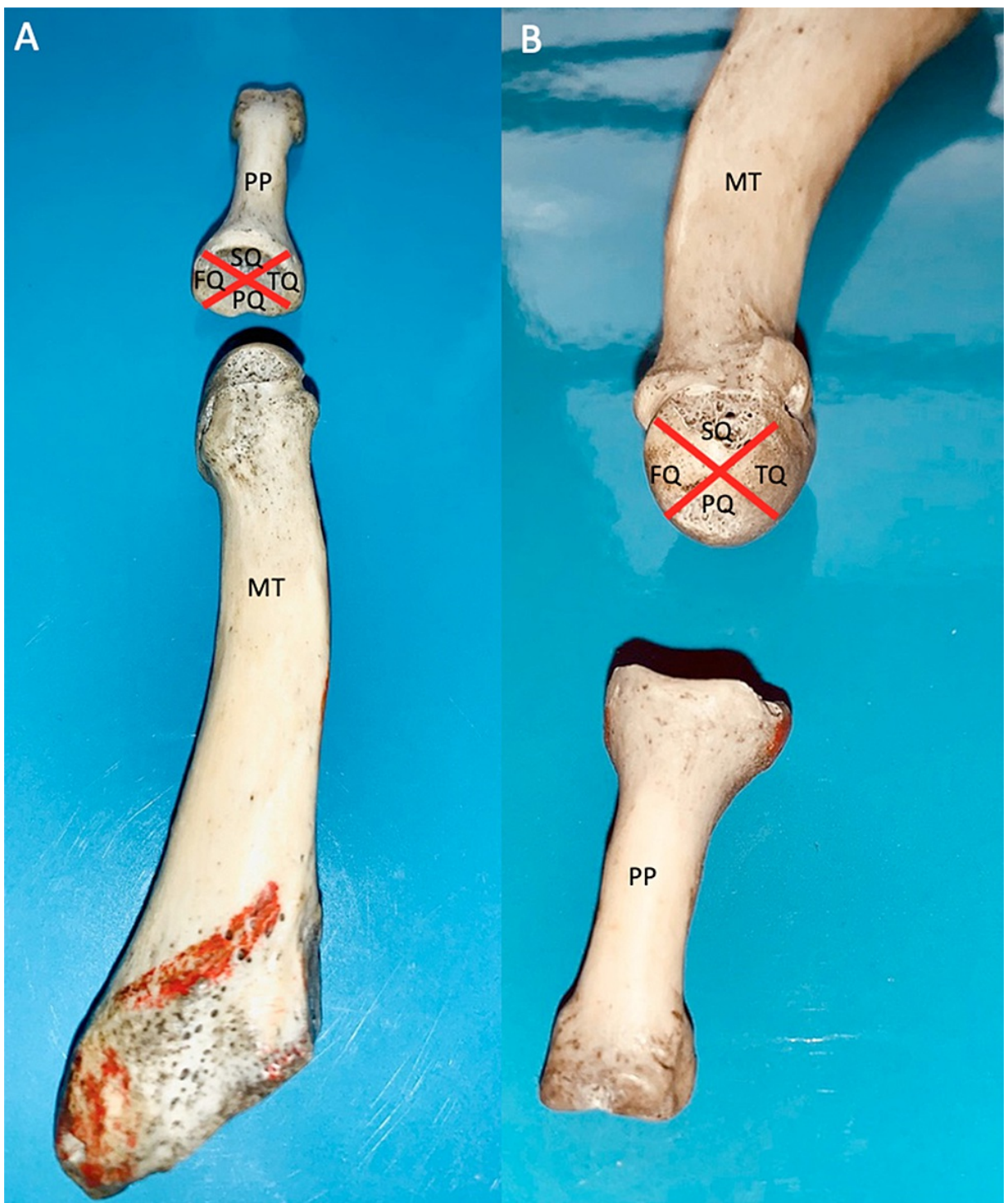

FIGURE 1: Representation of the quadrants of the metatarsophalangeal joint on the anatomical specimen.

(A) Division in quadrants of the proximal phalanx joint surface and (B) division in "pie-slice" quadrants of the metatarsal head joint surface. PP: proximal phalanx, M: metatarsal bone, SQ: superior quadrant; TQ: tibial quadrant (medial), PQ: plantar quadrant, FQ: fibular quadrant (lateral). 


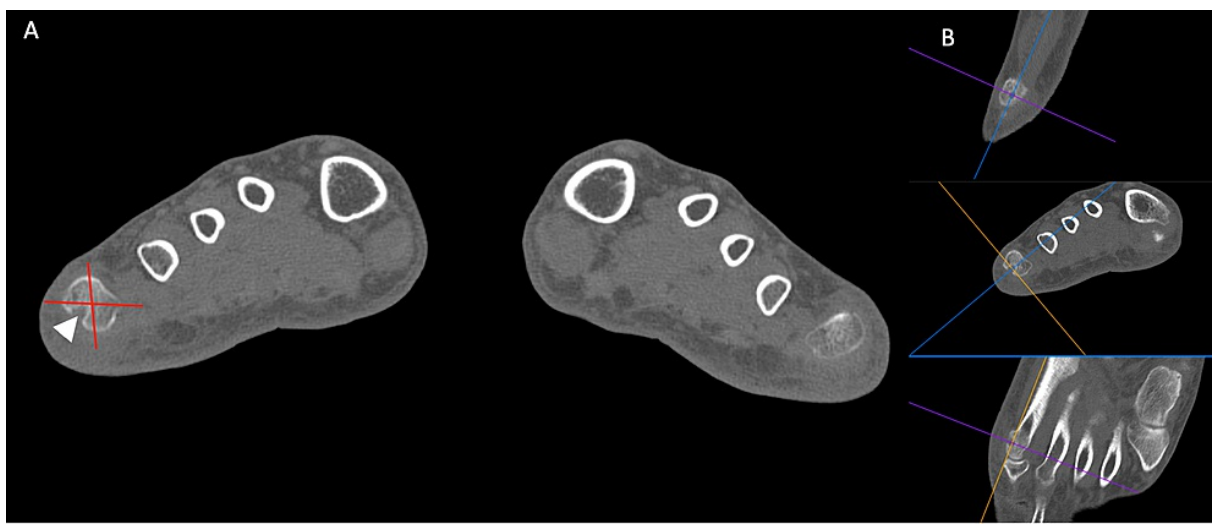

\section{FIGURE 2: Computed tomography of both forefeet with multi-planar reconstructions.}

(A) Bone erosion (white arrowhead) located at the fibular quadrant (FQ) of the fifth metatarsal head of the right foot (image acquired at $2 \mathrm{~mm}$ of thickness and reconstructed at $2 \mathrm{~mm}$ of thickness; (B) the multi-planar reconstruction (MPR) centered on the focal bone loss confirms the presence of the bone erosion. The red lines represent the division in quadrants.

Erosions larger than a quadrant were attributed to the quadrant in which the epicentre of the erosion was located. The epicentre of erosions was defined as the midpoint of the main axis of the erosion in the axial plane (perpendicular to the long axis of the metatarsal). If there was more than one erosion in a quadrant, we considered only the largest one. For both the MTH and PP, the analysis was extended to the first centimetre from the articular surface, as in previous studies [10].

Multi-level Method to Classify BE Topography

To analyze the anatomical distribution (topographical analysis) of BE, each erosion was classified according to the following multi-level method: (i) number of BE per patient; (ii) number of BE per side; (iii) number of $\mathrm{BE}$ on MTH; (iv) number of BE on proximal phalanges; (v) number of BE per quadrant.

\section{Statistical analysis}

The statistical analysis was carried out by a professional biostatistician (LS) using SAS software (SAS Institute, North Carolina, USA). Descriptive statistics (mean, standard deviation), t-test for paired and unpaired samples, Pearson's correlation coefficients, two-way analysis of variance (ANOVA 2) to compare the averages of the same sample, and a variance component analysis were carried out. The results were considered significant if $\mathrm{p}<0.05$.

\section{Results}

\section{Demographics and clinical features}

In the population of 18 patients, there were 12 women and 6 men. There was no significant difference in age between men and women $(64.2 \pm 10.7$ vs $61.8 \pm 17.0 ; \mathrm{p}=0.71)$. The mean disease duration was higher in women $(13.2 \pm 9.1$ years $)$ than in men $(5.5 \pm 4.7$ years; $p=0.027)$. At the clinical examination of the MTFJ, 9 out of 18 patients (50\%) were asymptomatic, 2 out of 18 (11.1\%) presented pain and/or of their left forefoot, 7 out of 18 (38.9\%) presented pain or swelling bilaterally.

Analysis of the Number of BE by the Multilevel Model

Number of BE per patient: In the population of 18 patients, CT revealed 324 erosions at the MTPJ. A total of $258 \mathrm{BE}$ were found at the MTH (80\%), while 66 were detected at the base of the proximal phalanx (20\%; $\mathrm{p}>0.0001$ ). The mean number of BE found on CT for each patient at the MTPJ (including both forefeet) was $18 \pm 7.8$. The number of $\mathrm{BE}$ in one forefoot was a poor predictor of the absolute number of $\mathrm{BE}$ of the contralateral one $(\mathrm{r}=0.54)$ even if the $\mathrm{BE}$ of the forefeet was statistically correlated in each patient $(\mathrm{p}=0.021)$. At ANOVA 2 analysis was no statistically significant correlation between the number of $\mathrm{BE}$ and demographic covariates such as patients' pain and swelling, age, gender, symptomatic/asymptomatic side(s), and the mean disease duration.

The number of erosions per side: There was no statistically significant difference between the cumulative number of $\mathrm{BE}$ of the right and left forefeet of all patients taken together (right forefoot $=167 \mathrm{BE}$ versus left forefoot $157=\mathrm{BE}, \mathrm{p}=0.7$ ). In addition, the mean number of $\mathrm{BE}$ considering the whole population was not 


\section{Cureus}

significantly different when comparing the left and the right forefoot (left forefoot $8.72 \pm 4.68$ versus right forefoot $9.28 \pm 4.28 ; \mathrm{p}=0.59$ ).

Number of BE at MTH: CT revealed a total of 258 BE at MTH (131 right sides versus 127 left sides; $\mathrm{p}=0.8$ ). Comparing the mean number of $\mathrm{BE}$ present between right and left forefoot revealed a statistically significant difference only for the fifth MTH. Indeed, the right side was more affected (mean number of erosions $1.34 \pm 1.2$ at the fifth right MTH versus $2.06 \pm 1.26$ at the left fifth MTH; mean difference $-0.67 \pm 1.19$; $\mathrm{p}=0.029)$. No statistically significant difference was found for the other MTH $(\mathrm{p}>0.05)$.

Number of BE at PP: CT revealed a total of $66 \mathrm{BE}$ at the base of the PP. There was no significant difference between the total number of $\mathrm{BE}$ on the right and the left forefeet (40 right forefeet versus 33 left forefeet, $\mathrm{p}=0.6$ ). In addition, the mean number of $\mathrm{BE}$ at the $\mathrm{PP}$ was not statistically significant when comparing the corresponding PP of each side ( $\mathrm{p}>0.05$ for all values). On the contrary, when comparing the PP of the same side, the number of $\mathrm{BE}$ of the $\mathrm{PP}$ was significantly different only when comparing the first $\mathrm{PP}(0.73 \pm 0.03)$ to the fifth PP of the right forefoot (mean difference $=0.02 \pm 0.07 ; \mathrm{p}=0.0038$ ).

Number of BE per quadrant: Table 1 details the BE number for each quadrant of each MTH and PP (first to fifth MTPJs) for the right and the left forefoot.

\begin{tabular}{|c|c|c|c|c|c|c|c|c|c|}
\hline $\begin{array}{l}\text { Number of } \\
\text { BE }\end{array}$ & $\begin{array}{l}\text { Superior } \\
\text { right }\end{array}$ & $\begin{array}{l}\text { Fibular } \\
\text { right }\end{array}$ & $\begin{array}{l}\text { Plantar } \\
\text { right }\end{array}$ & $\begin{array}{l}\text { Tibial } \\
\text { right }\end{array}$ & $\begin{array}{l}\text { Superior } \\
\text { left }\end{array}$ & $\begin{array}{l}\text { Fibular } \\
\text { left }\end{array}$ & $\begin{array}{l}\text { Plantar } \\
\text { left }\end{array}$ & $\begin{array}{l}\text { Tibial } \\
\text { left }\end{array}$ & Total \\
\hline $1^{\circ} \mathrm{MTH}$ & 5 & 9 & 10 & 13 & 5 & 11 & 8 & 9 & 70 \\
\hline $2^{\circ} \mathrm{MTH}$ & 3 & 1 & 4 & 7 & 2 & 9 & 7 & 6 & 39 \\
\hline $3^{\circ} \mathrm{MTH}$ & 1 & 7 & 5 & 14 & 2 & 7 & 5 & 5 & 46 \\
\hline $4^{\circ} \mathrm{MTH}$ & 4 & 5 & 3 & 6 & 3 & 4 & 7 & 12 & 44 \\
\hline $5^{\circ} \mathrm{MTH}$ & 5 & 13 & 11 & 8 & 3 & 7 & 7 & 8 & 62 \\
\hline $1^{\circ} \mathrm{PP}$ & 1 & 3 & 4 & 9 & 0 & 3 & 2 & 2 & 24 \\
\hline $2^{\circ} \mathrm{PP}$ & 1 & 0 & 3 & 4 & 1 & 0 & 1 & 2 & 12 \\
\hline $3^{\circ} \mathrm{PP}$ & 0 & 1 & 1 & 2 & 0 & 1 & 1 & 1 & 7 \\
\hline $4^{\circ} \mathrm{PP}$ & 0 & 1 & 1 & 3 & 1 & 1 & 1 & 2 & 10 \\
\hline $5^{\circ} \mathrm{PP}$ & 0 & 2 & 2 & 3 & 1 & 2 & 0 & 4 & 14 \\
\hline Total & 20 & 42 & 44 & 69 & 18 & 45 & 39 & 51 & 324 \\
\hline
\end{tabular}

TABLE 1: Absolute number of BE per side for each quadrant of each MTH and PP.

TOT: total.

Figure 3 represents the cumulative number of BE in each quadrant of the MTH and PP (first to fifth MTPIs) for the right and the left forefoot. 


\section{Cureus}
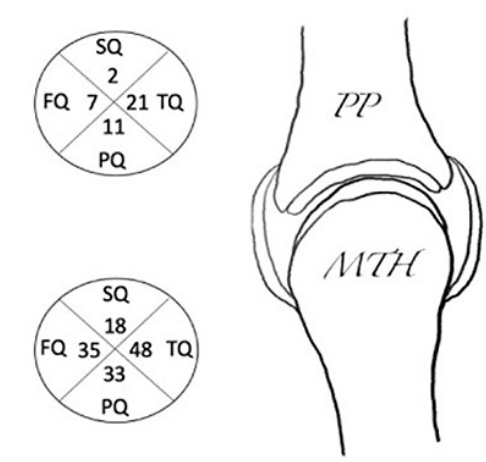

Right $\mathcal{M} \mathcal{T}$ PJ

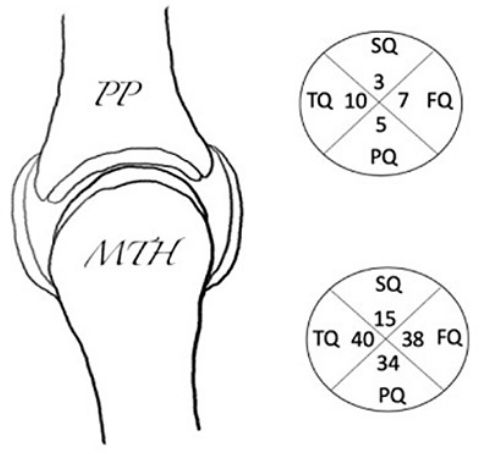

Left $M T^{\prime} P \mathcal{J}$

\section{FIGURE 3: Cumulative number of bone erosions.}

In each quadrant for the MTH and the PP for the right and the left sides with the MTPJ from the first to the fifth MTPJ were taken together. PP: proximal phalanx, MTH: metatarsal head, SQ: superior quadrant, TQ: tibial quadrant (medial), PQ: plantar quadrant, FQ: fibular quadrant (lateral).

Table 2 describes the cumulative number of BE, the percentage of BE in each quadrant for the MTH, the PP with the statistical values independently from the side, and the joint (MTPJ from first to fifth are taken together). On cumulative analysis, the superior quadrants were statistically less frequently affected than all other quadrants for both the MTH ( $>>0.0001)$ and the PP ( $<<0.001$; Table 2). On the contrary, the tibial quadrant showed a statistically significantly higher number of erosions compared to all other quadrants for the MTH $(\mathrm{p}<0.03)$, the PP $(\mathrm{p}<0.01)$, and for the MTPJ considered as a whole $(\mathrm{p}<0.0001)$. The plantar and the fibular quadrants were equally affected $(\mathrm{p}<0.05)$, both having more BE compared to the superior quadrants and less BE compared to the tibial quadrants (Table 2).

\begin{tabular}{|c|c|c|c|c|c|c|}
\hline Quadrant & $\begin{array}{l}\text { No. of BE } \\
\text { MTH }\end{array}$ & (\%) & $\begin{array}{l}\text { No. of BE } \\
\text { PP }\end{array}$ & (\%) & $\begin{array}{l}\text { No. of BE MTPJ } \\
\text { (MTH+PP) }\end{array}$ & (\%) \\
\hline SQ & 33 & $\begin{array}{l}(12,8 \%) \\
p>0.0001\end{array}$ & 5 & $(7.6 \%) ; p>0.001$ & 38 & $\begin{array}{l}(11.7 \%) \\
p<0.0001\end{array}$ \\
\hline FQ & 73 & $(28,3 \%) ; \mathrm{NS}$ & 13 & (19.7\%); NS & 86 & $(26.5 \%) ; \mathrm{NS}$ \\
\hline$P Q$ & 67 & (26\%); NS & 16 & (24.3\%); NS & 83 & (25.2\%);NS \\
\hline TQ & 85 & $(32,9 \%) ; p>0.003$ & 32 & $\begin{array}{l}(48,4 \%) \\
p>0.001\end{array}$ & 117 & $\begin{array}{l}(36.1 \%) \\
p>0.0001\end{array}$ \\
\hline $\begin{array}{l}\text { Total no. of } \\
\text { BE }\end{array}$ & 258 & $(100 \%)$ & 66 & $(100 \%)$ & 324 & (100\%) \\
\hline
\end{tabular}

\section{TABLE 2: Cumulative number of bone erosions for the metatarsal heads.}

PP and MTPJ independently from the side and the joint (MTPJ from first to fifth are taken together). The p-value is provided for quadrants with a statistically significantly different number of BE than the null hypothesis of a random distribution (i.e., $25 \%$ of erosion for each of quadrants). MTPJ: metatarsophalangeal joint, BE: bone erosions, MTH: metatarsal head, PP: proximal phalange, SQ: superior quadrant, FQ: fibular quadrant,

$\mathrm{PQ}$ : plantar quadrant; TQ: tibial quadrant, NS: no statistically significant difference than a random distribution of the bone erosion around the joint. 
The source of variance of the topography of BE was assessed considering the different levels of the analysis (Table 3).

\begin{tabular}{|c|c|c|c|c|}
\hline Variance component analysis & Variance components & SD & CV & CV (\%) \\
\hline Quadrant & 0.133 & 0.365 & 1.62 & 75.6 \\
\hline Joint & 0.036 & 0.190 & 0.844 & 20.5 \\
\hline Side & 0 & 0 & 0 & 0.0 \\
\hline Patient & 0.0069 & 0.083 & 0.369 & 3.9 \\
\hline Total & 0.174 & 0.420 & 1.87 & 100 \\
\hline
\end{tabular}

TABLE 3: Variance components analysis.

SD: standard deviation, CV: component of variance.

In the present study, a total of 324 positive quadrants for $\mathrm{BE}$ were identified in the 1140 quadrants; the proportion of $\mathrm{BE}$ is, therefore, $324 / 1440=0.225$ (i.d. $22.5 \%$ ). Therefore, being the percentage $\pi=0.225$, the total variance is noted as $\sigma^{2}$, being $\sigma^{2}=\pi \times(1-\pi)$. Therefore, in the present table, the total variance $\left(\sigma^{2}\right)$ is calculated as follows:

$\sigma^{2}=0.225 \times(1-0.225)=0.174$

The main factor contributing to the overall variance was the quadrant (explaining $75.6 \%$ variance). The variance calculated for the different MTPJ (e.g., the first, the second, etc.) accounted for $20.5 \%$ of the variance. Variance depending on the patient accounted only for $3.9 \%$ of the total variance. Finally, no significant variance depended on the side.

\section{Discussion}

The present study is the first to describe the location of BE at the MTPJ of both forefeet in patients with RA using a tomographic imaging technique, regardless of the predominantly symptomatic side. Previous studies assessing the location of $\mathrm{BE}$ at both forefeet were performed using radiographs and, thus, were limited by their bi-dimensional nature [16-18].

Unlike previous studies performed using MRI [10], we systematically mapped using isotropic CT images the BE topography at the MTFJ without focusing on the symptomatic but imaging both forefeet. Previously, only Boutry et al. [19] performed bilateral MRI in patients with RA, but they included the forefeet only in few selected cases, based on clinical symptoms.

In this study, we systematically assessed the topography of BE by dividing each joint surface into four quadrants, according to the scheme to map BE at the MCPJs used in previous studies using CT and HR-pQCT $[8,15]$. The analysis of 18 different patients resulted in the analysis of a total of 1440 quadrants (18 patient $\times$ 2 sides $\times 5$ MTFJ joints $\times 2$ joint surfaces $\times 4$ quadrants for each joint surface $=1440$ quadrants). This division of the joint surface allows counting the number of BE of distinct functional areas. At the MTFJ, the tibial and fibular aspects of the MTHs and PPs are subjected to the mechanical stress of the collateral ligaments at the enthesis. At the same time, the plantar areas of the joints are subject to mechanical stress due to body weight [8,10,19-22].

Our study provided some significant results. We observed that the absolute number of BE of a given forefoot is a poor predictor of the number of $\mathrm{BE}$ occurring on the contralateral side. This finding is significant because bilateral CT has been suggested as a reliable technique to quantify BE [12]. CT scoring for BE in RA was recently used, applying the OMERACT MRI scoring system to CT, focusing on the more symptomatic foot. However, a recent meta-analysis on the validity of the RA MRI score applied to the more symptomatic forefoot using the OMERACT filter concluded that even when using MRI, the OMERACT score is not related to all pathologic findings, including BE. Our study suggests that both forefeet should be assessed for a reliable evaluation of the $\mathrm{BE}$.

Second, no difference in terms of the number of BE was found between the right and left sides. This result suggests that $\mathrm{BE}$ topography is not correlated to side dominance, largely favouring the right side in the general population. These results are in keeping with the results obtained by Yaku et al. [18], who studied the effect of handedness in joint involvement in patients with RA on radiographs. The authors assessed 28 different joints in 334 patients, including the MTPJ, and found no significant effect of handedness in 
Third, there is a statistically significantly higher number of BE at the MTH compared to PP (258 vs 66). These data parallel previous MRI studies $[10,19]$ reporting a higher number of BE at the MTH of the more symptomatic forefoot. Our results corroborate the hypothesis that the weight-bearing load on the MTH may induce a higher occurrence of BE compared to PP, given the higher mechanical demand.

Fourth, in our population, only the fifth MTH of both sides and the first proximal phalanx of the right side were more affected compared to their, respectively, paired joint surfaces. This finding is similar to results from previous studies using radiographs in which a higher rate of BE was observed at first and the fourth MTFJ [4] and at the fifth MTFJ [19,23-25].

Finally, BE was statistically more frequent at the tibial quadrant for both the MTH and PP in our population. The fibular and plantar quadrant were statistically more affected by BE than the superior quadrant. Our results agree with previous radiographic and sonographic investigations reporting the highest number of $\mathrm{BE}$ at the tibial aspects of the MTH $[24,26,27]$.

However, these data disagree with the findings of Siddle et al. [10], reporting an absolute predominance of $\mathrm{BE}$ at the plantar aspect of the MTFJ. This discordance could be explained by the different definitions of the joint areas. In their study, the joint surface was divided into four areas by a vertical and horizontal line, making it difficult to attribute BE to the plantar or the lateral aspects of the joint. Even if most of the mechanical load is located at the plantar aspect of the MTFJ, stabilized by the plantar plate [28], our study suggests that the mechanical demand at the insertion of the collateral ligaments may favour the location of $\mathrm{BE}$ at the tibial and fibular aspect of the joints.

The biomechanical demand of the collateral ligaments at the MTPJ [18,28], along with that of the intermetatarsal "tie-bar" ligamentous system [18], is well established [28]. The higher rate of BE at the tibial aspect of the MTFJ lasts partially unexplained. Other factors such as the distribution of cortical microchannels (CoMiCs) or plantar plate or capsule failure could determine BE's prevalent location at the tibial aspect of the MTFJ. Other studies using HR-pQCT for CoMiCs [15] or MRI to assess the integrity of the bone plate and collateral ligaments should be carried out to test these hypotheses.

The present study has several limitations. The population included in this study is limited to 18 patients. Our number of patients is like previous studies performed using CT or MRI on a single foot [10,19]. Multicentric studies should be performed to obtain a larger extent of data considering the high number of parameters considered in the statistical analysis. In addition, we did not consider in our analysis laboratory test results such as the C-reactive protein (CRP) level or the Disease Activity Score (DAS) used in clinical practice. However, previous studies showed no correlation between the overall disease activity, local clinical symptoms at MTPJ, and BE [10].

Furthermore, we did not consider as covariates the presence of factors favouring the onset of BE, including joint subluxation and bone plate failure, because these findings are difficult to detect or questionable on CT images performed in a non-weight-bearing supine position. Concomitant deformities of the foot [25] and capsular and plantar plate failure [10], modifying the area of higher mechanical demand at the forefeet were suggested as possible factors to determine the topography of BE in MTFJs in different populations of patients. Finally, only the topography of the largest BE for each joint surface was considered in the analysis. The topography of small size BE could have a different spatial pattern of anatomical location.

\section{Conclusions}

The CT assessment of both forefeet showed that $\mathrm{BE}$ were more commonly found at the tibial aspect of the MTFJ, mostly at the MTH. The plantar and fibular aspect of the MTFJ had a lower rate of BE, with a similar incidence, suggesting that the biomechanical demand of the collateral ligaments at the MTPJ may play a key role in determining the topography of $\mathrm{BE}$ at the forefeet.

\section{Additional Information}

\section{Disclosures}

Human subjects: Consent was obtained or waived by all participants in this study. Comité d' Etique CHU de Liège issued approval B707201419709. The Local Ethics Committee approved this retrospective study (Belgian national number of Ethic board approval: number B707201419709), and patients' written consensus was waived for this retrospective study. Animal subjects: All authors have confirmed that this study did not involve animal subjects or tissue. Conflicts of interest: In compliance with the ICMJE uniform disclosure form, all authors declare the following: Payment/services info: All authors have declared that no financial support was received from any organization for the submitted work. Financial relationships: All authors have declared that they have no financial relationships at present or within the previous three years with any organizations that might have an interest in the submitted work. Other relationships: All authors have declared that there are no other relationships or activities that could appear to have influenced the 


\section{References}

1. Aletaha D, Neogi T, Silman AJ, et al.: 2010 Rheumatoid arthritis classification criteria: an American College of Rheumatology/European League Against Rheumatism collaborative initiative. Arthritis Rheum. 2010, 62:2569-81. 10.1002/art.27584

2. Myasoedova E, Davis J, Matteson EL, Crowson CS: Is the epidemiology of rheumatoid arthritis changing? Results from a population-based incidence study, 1985-2014. Ann Rheum Dis. 2020, 79:440-4. 10.1136/annrheumdis-2019-216694

3. Schett G, Gravallese E: Bone erosion in rheumatoid arthritis: mechanisms, diagnosis and treatment . Nat Rev Rheumatol. 2012, 8:656-64. 10.1038/nrrheum.2012.153

4. Otter SJ, Lucas K, Springett K, et al.: Foot pain in rheumatoid arthritis prevalence, risk factors and management: an epidemiological study. Clin Rheumatol. 2010, 29:255-71. 10.1007/s10067-009-1312-y

5. van der Heijde DM, van Leeuwen MA, van Riel PL, Koster AM, van 't Hof MA, van Rijswijk MH, van de Putte LB: Biannual radiographic assessments of hands and feet in a three-year prospective followup of patients with early rheumatoid arthritis. Arthritis Rheum. 1992, 35:26-34. 10.1002/art.1780350105

6. McGonagle D: The history of erosions in rheumatoid arthritis: are erosions history? . Arthritis Rheum. 2010, 62:312-5. 10.1002/art.27199

7. Sudoł-Szopińska I, Jurik AG, Eshed I, et al.: Recommendations of the ESSR Arthritis Subcommittee for the use of magnetic resonance imaging in musculoskeletal rheumatic diseases. Semin Musculoskelet Radiol. 2015, 19:396-411. 10.1055/s-0035-1564696

8. McGonagle D, Tan AL, Møller Døhn U, Ostergaard M, Benjamin M: Microanatomic studies to define predictive factors for the topography of periarticular erosion formation in inflammatory arthritis. Arthritis Rheum. 2009, 60:1042-51. 10.1002/art.24417

9. Martel W, Hayes JT, Duff IF: The patterns of bone erosion in the hand and wrist in rheumatoid arthritis . Radiology. 1965, 84:204-14. 10.1148/84.2.204

10. Siddle HJ, Hensor EM, Hodgson RJ, Grainger AJ, Redmond AC, Wakefield RJ, Helliwell PS: Anatomical location of erosions at the metatarsophalangeal joints in patients with rheumatoid arthritis. Rheumatology (Oxford). 2014, 53:932-6. 10.1093/rheumatology/ket478

11. Yaku A, Hashimoto M, Furu M, et al.: Relationship between handedness and joint involvement in rheumatoid arthritis. Sci Rep. 2016, 6:39180. 10.1038/srep39180

12. Ørnbjerg LM, Østergaard M: Assessment of structural damage progression in established rheumatoid arthritis by conventional radiography, computed tomography, and magnetic resonance imaging. Best Pract Res Clin Rheumatol. 2019, 33:101481. 10.1016/j.berh.2019.101481

13. Simoni P, Gérard L, Kaiser MJ, Ribbens C, Rinkin C, Malaise O, Malaise M: Use of tomosynthesis for detection of bone erosions of the foot in patients with established rheumatoid arthritis: comparison with radiography and CT. AJR Am J Roentgenol. 2015, 205:364-70. 10.2214/AJR.14.14120

14. Døhn UM, Ejbjerg BJ, Hasselquist M, et al.: Rheumatoid arthritis bone erosion volumes on CT and MRI: reliability and correlations with erosion scores on CT, MRI and radiography. Ann Rheum Dis. 2007, 66:138892. 10.1136/ard.2007.072520

15. Døhn UM, Terslev L, Szkudlarek M, et al.: Detection, scoring and volume assessment of bone erosions by ultrasonography in rheumatoid arthritis: comparison with CT. Ann Rheum Dis. 2013, 72:530-4. 10.1136/annrheumdis-2011-201114

16. Werner D, Simon D, Englbrecht M, et al.: Early changes of the cortical micro-channel system in the bare area of the joints of patients with rheumatoid arthritis. Arthritis Rheumatol. 2017, 69:1580-7. 10.1002/art.40148

17. Van der Heijden KW, Rasker JJ, Jacobs JW, Dey K: Kates forefoot arthroplasty in rheumatoid arthritis. A 5year follow up study. J Rheumatol. 1992, 19:1545-50.

18. Buchbender C, Scherer A, Miese F, et al.: Patterns of magnetic resonance imaging of the foot in rheumatoid arthritis: which joints are most frequently involved?. Rheumatol Int. 2013, 33:1731-6. 10.1007/s00296-0122648-1

19. Boutry N, Flipo RM, Cotten A: MR imaging appearance of rheumatoid arthritis in the foot . Semin Musculoskelet Radiol. 2005, 9:199-209. 10.1055/s-2005-921940

20. Siddle HJ, Hodgson RJ, O'Connor P, et al.: Magnetic resonance arthrography of lesser metatarsophalangeal joints in patients with rheumatoid arthritis: relationship to clinical, biomechanical, and radiographic variables. J Rheumatol. 2012, 39:1786-91. 10.3899/jrheum.120392

21. van der Leeden M, Steultjens MP, Ursum J, Dahmen R, Roorda LD, Schaardenburg DV, Dekker J: Prevalence and course of forefoot impairments and walking disability in the first eight years of rheumatoid arthritis. Arthritis Rheum. 2008, 59:1596-602. 10.1002/art.24188

22. Dakkak YJ, van der Heijde DM, Reijnierse M, van der Helm-van Mil AH: Validity of the rheumatoid arthritis MRI score applied to the forefeet using the OMERACT filter: a systematic literature review. RMD Open. 2018, 4:e000796. 10.1136/rmdopen-2018-000796

23. Resnick D: Diagnosis of Bone and Joint Disorders . WB Saunders, Philadelphia; 2002.

24. Tuna H, Birtane M, Taştekin N, Kokino S: Pedobarography and its relation to radiologic erosion scores in rheumatoid arthritis. Rheumatol Int. 2005, 26:42-7. 10.1007/s00296-004-0504-7

25. Boutroy S, Bouxsein ML, Munoz F, Delmas PD: In vivo assessment of trabecular bone microarchitecture by high-resolution peripheral quantitative computed tomography. J Clin Endocrinol Metab. 2005, 90:6508-15. 10.1210/jc.2005-1258

26. Lopez-Ben R, Bernreuter WK, Moreland LW, Alarcon GS: Ultrasound detection of bone erosions in rheumatoid arthritis: a comparison to routine radiographs of the hands and feet. Skeletal Radiol. 2004, 33:80-4. 10.1007/s00256-003-0693-2

27. Suero EM, Meyers KN, Bohne WH: Stability of the metatarsophalangeal joint of the lesser toes: a cadaveric study. J Orthop Res. 2012, 30:1995-8. 10.1002/jor.22173 


\section{Cureus}

28. Wang B, Guss A, Chalayon O, Bachus KN, Barg A, Saltzman CL: Deep transverse metatarsal ligament and static stability of lesser metatarsophalangeal joints: a cadaveric study. Foot Ankle Int. 2015, 36:573-8. $10.1177 / 1071100714563310$ 